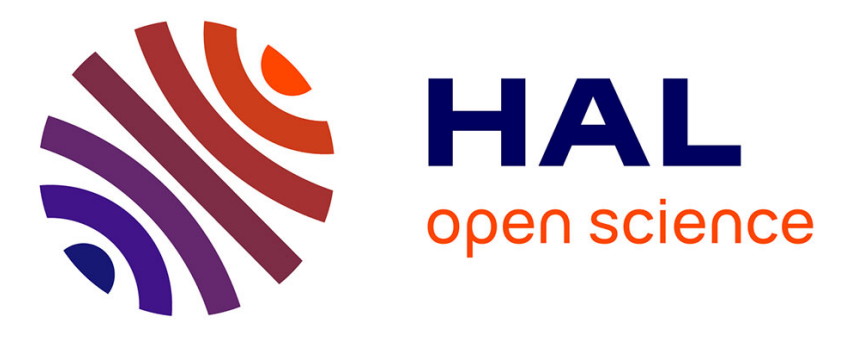

\title{
Singlet fission in naturally-organized carotenoid molecules
}

\author{
Annamaria Quaranta, Anja Krieger-Liszkay, Andrew Pascal, François \\ Perreau, Bruno Robert, Mikas Vengris, Manuel J. Llansola-Portoles
}

\section{To cite this version:}

Annamaria Quaranta, Anja Krieger-Liszkay, Andrew Pascal, François Perreau, Bruno Robert, et al.. Singlet fission in naturally-organized carotenoid molecules. Physical Chemistry Chemical Physics, 2021, 23 (8), pp.4768-4776. 10.1039/D0CP04493H . hal-03282317

\section{HAL Id: hal-03282317 https://hal.science/hal-03282317}

Submitted on 13 Jul 2021

HAL is a multi-disciplinary open access archive for the deposit and dissemination of scientific research documents, whether they are published or not. The documents may come from teaching and research institutions in France or abroad, or from public or private research centers.
L'archive ouverte pluridisciplinaire HAL, est destinée au dépôt et à la diffusion de documents scientifiques de niveau recherche, publiés ou non, émanant des établissements d'enseignement et de recherche français ou étrangers, des laboratoires publics ou privés. 


\title{
Singlet Fission in Naturally-Organized Carotenoid Molecules
}

\author{
Annamaria Quaranta ${ }^{\dagger}$, Anja Krieger-Liszkay ${ }^{\dagger}$, Andrew A. Pascal ${ }^{\dagger}$, François Perreau ${ }^{\ddagger}$, Bruno Rob- \\ ert $^{\dagger}$, Mikas Vengris ${ }^{\S *}$ and Manuel J. Llansola-Portoles ${ }^{\dagger *}$ \\ † Université Paris-Saclay, CEA, CNRS, Institute for Integrative Biology of the Cell (I2BC), 91198, Gif-sur-Yvette, \\ France \\ ‡ Institut Jean-Pierre Bourgin, INRAE, AgroParisTech, Université Paris-Saclay, 780oo, Versailles, France \\ $\S$ Laser Research Center, Faculty of Physics, Vilnius University, Sauletekio Ave. 10, LT-10223, Vilnius, Lithuania
}

Keywords: singlet exciton fission, daffodil chromoplast, aggregated carotenoids, triplet state

\begin{abstract}
We have investigated the photophysics of aggregated lutein/violaxanthin in daffodil chromoplasts. We reveal the presence of three carotenoid aggregate species, the main one composed of a mixture of lutein/violaxanthin absorbing at $481 \mathrm{~nm}$, and two secondary populations of aggregated carotenoids absorbing circa 500 and $402 \mathrm{~nm}$. The major population exhibits an efficient singlet fission process, generating $\mu$ s-lived triplet states on an ultrafast timescale. The structural organization of aggregated lutein/violaxanthin in daffodil chromoplasts produces well-defined electronic levels that permit the energetic pathways to be disentangled unequivocally, allowing us to propose a consistent mechanism for singlet fission in carotenoid aggregates. Transient absorption measurements on this system reveal for the first time an entangled triplet signature for carotenoid aggregates, and its evolution into dissociated triplet states. A clear picture of the carotenoid singlet fission pathway is obtained, which is usually blurred due to the intrinsic disorder of carotenoid aggregates.
\end{abstract}

\section{INTRODUCTION}

The bright colours of many flowers and fruits originate from the carotenoids accumulated in specialized plastids in the plant cell, termed chromoplasts. Chromoplasts are generally produced in flower petals and ripe fruit through a remodeling of the photosynthetic chloroplast ${ }^{1}$, involving chlorophyll degradation, down-regulation of genes encoding for photosynthetic proteins, and up-regulation of stress-response and carotenoid-synthesis genes (although differentiation may also occur from other plastid types²). In mature chromoplasts, the carotenoid pigments are sequestered in the form of crystal-like structures (crystalloids), granules, or stromal plastoglobules 3 . The main function of the chromoplast is thought to be secondary signaling, attracting animals to flowers and fruits to aid pollination and seed dispersal, respectively. They are also the site of protein, lipid and carbohydrate biosynthesis ${ }^{4}$, and play an important role in fruit and flower scent (for instance, one of the main components of the tomato scent is a degradation product of chromoplastic lycopene) $)^{5}$. It is interesting to note that the chloroplast/chromoplast transition is in some cases a reversible process, such that chromoplasts can re-acquire photosynthetic activity and differentiate back into chloroplasts ${ }^{1,2}$.

Carotenoid molecules are tetraterpenoid derivatives formed by eight isoprene units. They display a wide variety of functions in photosynthetic tissues, some of which are also observed throughout nature. They play an important role in harvesting photons in the blue-green range in photosynthesis ${ }^{6}$, and ensure efficient photoprotection of the photosynthetic apparatus - through the quenching of chlorophyll triplet ${ }^{7}$ and singlet ${ }^{8}$ excited states, and by short-circuiting lipid peroxidation'. Carotenoids exhibit strong electronic absorption corresponding to a transition from the ground state to the second excited state $\left(S_{0} \rightarrow S_{2}\right)$, while transition to the first excited state (which has the same symmetry as the ground state) is one-photon forbidden ${ }^{10}$. After photoexcitation, isolated carotenoids decay rapidly to the ground state in a few picoseconds ${ }^{11,12}$. Although chromoplasts are not expected to display any specific functional photoactivity, we recently discovered that the lycopene crystalloids in tomato chromoplasts do indeed exhibit photophysics that are not observed in isolated carotenoids ${ }^{13}$. This self-associated lycopene dissipates excitation energy via singlet fission, a mechanism we tentatively proposed to be photoprotective. In the singlet exciton fission process, one singlet excited state splits into two excited triplets $\left(\mathrm{T}_{1}+\mathrm{T}_{1}\right)$, fulfilling the energetic condition $\mathrm{E}\left(\mathrm{S}_{1}\right) \geq$ $2 \bullet E\left(T_{1}\right)^{14-16}$. Carotenoids in tightly-interacting assemblies in vitro have been shown to generate triplets by singlet fission - such as aggregated astaxantin ${ }^{17,18}, \beta$-carotene ${ }^{19,20}$, and 
zeaxanthin ${ }^{21-23}$ - but up to now, tomato chromoplasts represent the only known example of this process in a biological structure ${ }^{13}$.

We investigated the lutein/violaxanthin photochemistry in daffodil <Narcissus pseudonarcissus L. $>$ chromoplasts $^{24}$, ${ }^{25}$, to determine whether singlet fission is unique to the lycopene in tomato crystalloids, or is a more general chromoplast phenomenon. Daffodil chromoplasts accumulate these carotenoids in a concentric membrane system, forming condensed structures involving carotenoids and acyl lipid molecules (they belong to the class of so-called membranous chromoplasts) ${ }^{24},{ }^{25}$. These structures allow close interactions between carotenoids, which perturb the absorption properties of lutein and violaxanthin. We show that singlet fission indeed occurs in these plastids, suggesting that this process may be common to all carotenoidcontaining chromoplasts. Our experiments reveal the singlet fission mechanism in unprecedented detail. The information on specific energy levels of intermediate species allows us to identify the entangled triplet pair, and to pinpoint the spectral signatures of the disentangled triplet excited state(s) of the excitonic lutein/violaxanthin system.

\section{MATERIALS AND METHODS}

Chromoplasts of the inner coronae of fully-opened daffodil flowers (Narcissus pseudonarcissus L. cv Dutch Master), at developmental stage $\mathrm{IV}^{26}$, were isolated according to the method of Liedvogel et. al. ${ }^{25}$ using a step gradient in which chromoplasts accumulate at the $15 \% / 30 \%$ sucrose interphase ${ }^{27}$. The isolated chromoplasts were suspended in $100 \mathrm{mM}$ Tris/ $\mathrm{HCl} \mathrm{pH} \mathrm{7.4,} 10 \mathrm{mM} \mathrm{MgCl}$, $1 \mathrm{mM}$ 1,4-dithioerythritol. The preparations were stored at $-80^{\circ} \mathrm{C}$.

For pigment determination, the preparation was centrifuged, the supernatant discarded and the pelleted chromoplasts were resuspended in $2 \mathrm{~mL}$ acetone. $5 \mu \mathrm{L}$ was then subjected to liquid chromatography at a flow rate of $0.5 \mathrm{~mL}$ $\mathrm{min}^{-1}$, using an HPLC column (Uptisphere Strategy C18-HQ $250 \times 3 \mathrm{~mm} 3 \mu \mathrm{m}$, Interchim, Montluçon, France) with a liquid chromatography chain HPLC/UV (Shimadzu, Tokyo, Japan) which included two pumps (LC-2oAD), a sample manager (SIL-2oAC HT), a column oven (CTO-2oA) and a UV diode-array detector (UVSPD-M2oA) monitoring the absorbance at $450 \mathrm{~nm}$. Mobile phase A was composed of $10 \%$ water and $90 \%$ acetonitrile with $0.5 \%$ acetic acid, mobile phase B of ethyl acetate containing $0.5 \%$ acetic acid, and the elution gradient was as follows: 1 min $90 \% \mathrm{~A}$ : $10 \% \mathrm{~B}$, then a linear gradient to $5 \% \mathrm{~A}: 95 \% \mathrm{~B}$ after 25 min. Concentrations of each pigment were calculated based on their peak areas, calibrated using injected standards or (for some minor pigments) with known absorption factors for each compound at $450 \mathrm{~nm}$.

Absorption spectra were measured using a Varian Cary E5 scanning spectrophotometer. Low temperature measurements were performed in a helium bath cryostat (Maico Metriks, Tartu, Estonia); $60 \%$ glycerol (v/v) was added to the sample prior to freezing.

Circular dichroism spectra were recorded on an Aviv 215 spectropolarimeter (Aviv Biomedical). Near-UV CD spectra were recorded between 300 and $600 \mathrm{~nm}$ using a square cell with a $1 \mathrm{~cm}$ path length, and an averaging time of $1 \mathrm{~s}$ per step. Three consecutive scans from each sample were merged to produce an averaged spectrum, which was then corrected against buffer baselines measured under the same conditions. Dichroism value is measured in mdeg.

Resonance Raman spectra were recorded at $77 \mathrm{~K}$ with laser excitations obtained from Coherent Ar+ (Sabre) and $\mathrm{Kr}^{+}$(Innova 90) lasers. Output laser powers of 10-100 $\mathrm{mW}$ were attenuated to $<5 \mathrm{~mW}$ at the sample. Scattered light was collected at $90^{\circ}$ to the incident light, and focused into a Jobin-Yvon U1ooo double-grating spectrometer (180o grooves $/ \mathrm{mm}$ ) equipped with a red-sensitive, back-illuminated, $\mathrm{LN}_{2}$-cooled CCD camera. The sample stability and integrity were assessed based on the stability of the Raman signal.

Nano-to-millisecond transient absorption experiments were performed on an Edinburgh Instruments LP92o Flash Photolysis Spectrometer system, incorporating a Continuum Surelite OPO. The OPO was pumped by a Q-switched $\mathrm{Nd}$ :YAG laser operating at $355 \mathrm{~nm}$. The LP920 system is equipped with a $450 \mathrm{~W}$ Xenon arc lamp as the probe for the transient absorption measurements; this lamp was pulsed in the time range $10 \mathrm{~ns}$ to $100 \mu \mathrm{s}$. Detection in the LP920 system was performed either via a 500-nm-blazed Czerny-Turner monochromator (bandwidth 1-5 nm) coupled with a Hamamatsu R928 photomultiplier tube (kinetics mode), or via a 500-nm-blazed spectrograph (bandwidth $5 \mathrm{~nm}$ ) coupled with a water-cooled ICCD nanosecond Andor DH720 camera (spectral mode). Excitation energy was approximately $15 \mathrm{~mJ} /$ pulse.

Femto-to-nanosecond time-resolved transient absorption spectra were recorded on a setup described in detail elsewhere ${ }^{28}$. Pump and probe pulses were derived from an amplified Ti:Sapphire laser (Libra, Coherent). Tunable pump pulses with center wavelength at $490 \mathrm{~nm}$ were obtained from an optical parametric amplifier (Topas, Light Conversion). White light continuum generated in $\mathrm{CaF}_{2}$ from the fundamental output of the laser was used to probe the absorption changes in the $350-750 \mathrm{~nm}$ range. The time resolution of the instrument is ca. $\mathbf{1 2 0} \mathrm{fs}$. Excitation energies were set to approximately $200 \mathrm{~nJ} /$ pulse.

\section{RESULTS AND DISCUSSION}

The carotenoid content of daffodil chromoplasts was determined by HPLC (Supporting information, Figure S1). The carotenoids with significant concentrations are lutein and violaxanthin, with a ratio circa 5:4. Lutein and violaxanthin in cyclohexane both present the typical absorption spectrum for carotenoids, with four bands corresponding to the vibronic sublevels o-3, o-2, o-1, and o-o of the $\mathrm{S}_{\mathrm{o}} \rightarrow \mathrm{S}_{2}$ electronic transition - at 395, 416, 440 \& $474 \mathrm{~nm}$ and 393, $422,452 \& 471 \mathrm{~nm}$, respectively (Fig. 1a) ${ }^{29}$. Figure $1 \mathrm{~b}$ shows the absorption spectrum of broken daffodil chromoplasts in $99 \%$ ethanol, which disrupts the chromoplast and solubilizes the carotenoids. This spectrum, with maxima at $394,418,442 \& 471 \mathrm{~nm}$, is essentially identical to a linear combination of isolated lutein and violaxanthin using the proportion obtained from the HPLC analysis (supporting information, Figure S2). The absorption spectrum of intact 
chromoplasts at room temperature exhibits broad vibronic transitions peaking at 393, 422, 447 and $481 \mathrm{~nm}$ (Fig. 1C). The $\mathrm{o}-\mathrm{o}$ band at $481 \mathrm{~nm}$ is redshifted relative to the spectrum of solubilized chromoplasts (note that the absorption of lutein may be observed as high as $485 \mathrm{~nm}$ in high polarizability solvents ${ }^{30}$ ). The intensity of the $0-3$ and $0-2$ electronic transitions is significantly higher in chromoplasts than in monomeric carotenoids (cf. Fig. 1a,b,c), which may indicate some perturbation of the electronic transition caused by packing interactions ${ }^{31}$. The absorption spectrum of daffodil chromoplasts recorded at cryogenic temperature $(4.2 \mathrm{~K})$ reveals a complex picture, indicating the presence of several carotenoid populations (Fig. 1d) which can be resolved by second derivative analysis (Fig. 1e). The red part of the absorption spectrum shows the presence of two species with close electronic transitions: a dominant species (species 1) absorbing at 422, $451 \& 487 \mathrm{~nm}$, and a minor one (species 2) with absorption at $430,463 \& 500 \mathrm{~nm}(\mathrm{o}-2$, o-1 \& $0-0$, respectively). An additional band is present at $402 \mathrm{~nm}$ whose second derivative shows no clear structure. This suggests that this $402-\mathrm{nm}$ band is not a $0-3$ vibronic transition of species $1 \& 2$, but could rather arise from a third, blue-shifted carotenoid population (species 3). Overall, the absorption at $4.2 \mathrm{~K}$ presents a characteristic bathochromic shift relative to the spectrum at room temperature $^{32,33}$ (note red-shift of the major o-o band from 481 to $487 \mathrm{~nm}$ ). The circular dichroism of daffodil chromoplasts at room temperature (Fig if) exhibits chirality across the whole spectral range, with a dominant S-shaped feature on the blue side of the envelope (maximum $397 \mathrm{~nm}$, minimum $375 \mathrm{~nm}$ ). The CD spectrum of solubilized chromoplasts displays no chirality (data not shown). The observed chirality for intact chromoplasts indicates that all three species are constituted from aggregated (interacting) carotenoid molecules. Species 3 has a blue shifted o-o transition, around $402 \mathrm{~nm}$ at $4.2 \mathrm{~K}$, and displays a large CD signature. This is representative of $\mathrm{H}$-aggregates, where exciton coupling is strong and extends over multiple chromophores. The absorption of such strongly-coupled $\mathrm{H}$-aggregates collapses into a single electronic transition, with the large hypsochromic shift observed here (circa $5000 \mathrm{~cm}^{-1}$ relative to monomeric violaxanthin and lutein) ${ }^{34}$. Species 1 \& 2 conserve the vibronic peaks of monomeric carotenoids, and exhibit only a small bathochromic shift relative to them. Carotenoid aggregates that have a predominant bathochromic shift have been traditionally interpreted as J-aggregates $35,{ }^{36}$. However, species $1 \& 2$ do not resemble the typical narrow exciton-coupled bands expected for strongly-coupled J-aggregates ${ }^{17,} 18,37$. Their absorption and CD spectra more closely resemble model spectra of weaklycoupled H-aggregates of lutein ${ }^{31}$. Further studies are necessary to determine the precise molecular interaction(s) involved in species 1 \& 2 .
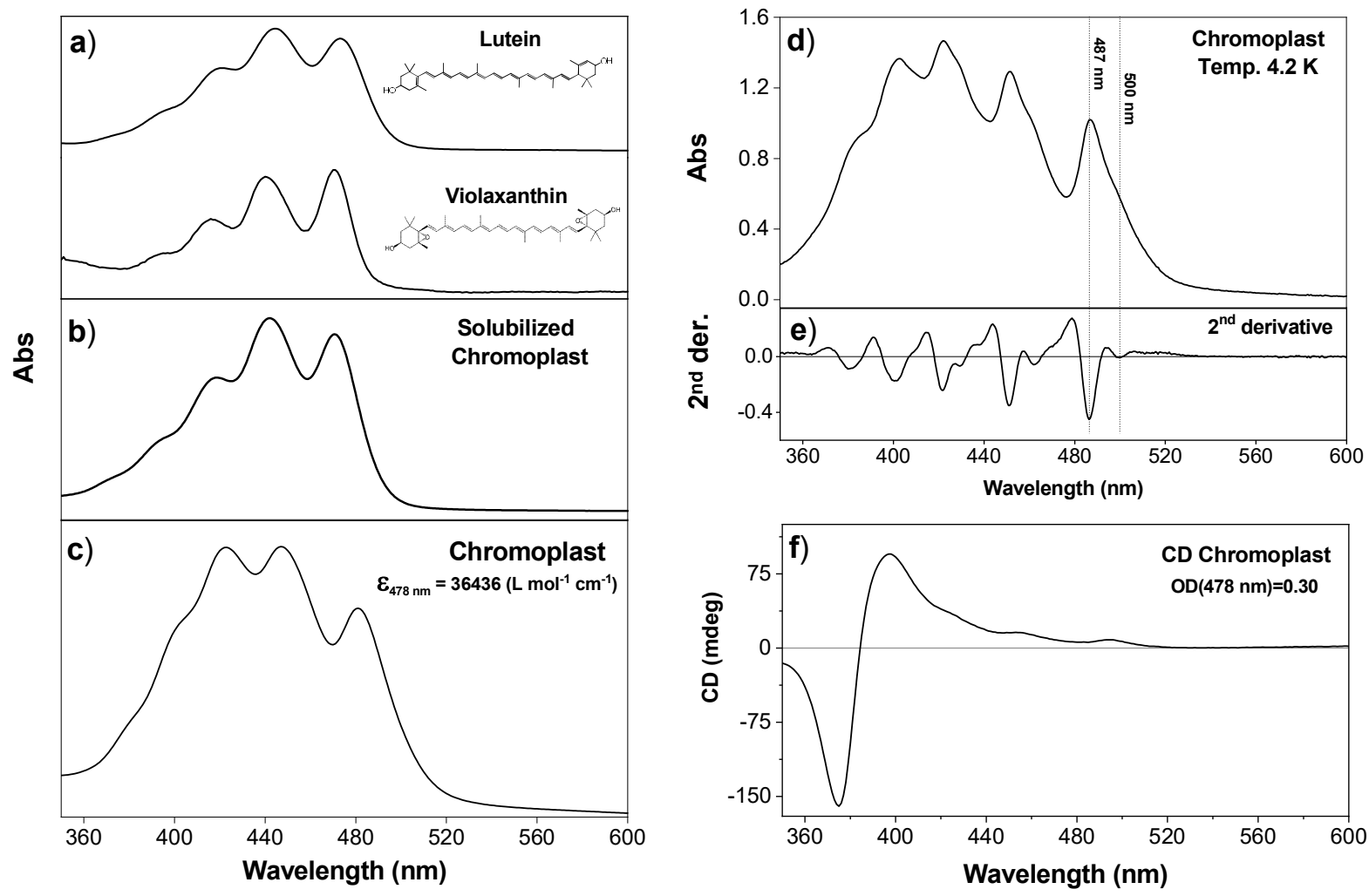

Figure 1: Room temperature absorption spectra of (a) monomeric lutein and violaxanthin in n-hexane; (b) solubilized chromoplasts; and (c) intact daffodil chromoplasts in buffer solution. (d) $4.2 \mathrm{~K}$ absorption spectrum of daffodil chromoplasts, and (e) its second derivative. (f) CD spectrum of daffodil chromoplasts in buffer solution, obtained with 0.3 OD at $478 \mathrm{~nm}$. 
We used resonance Raman spectroscopy ${ }^{38}$ at three different excitation wavelengths to characterize the three carotenoid species in daffodil chromoplasts. The high selectivity of this technique provides information for pigment populations individually, by exciting close to their respective absorption maxima. Figure 2 shows the resonance Raman spectra at $77 \mathrm{~K}$ for isolated all-trans lutein and violaxanthin in cyclohexane, measured at $488.0 \mathrm{~nm}$, and for intact chromoplasts in buffer excited at 488.0, 501.7 and $406.7 \mathrm{~nm}$. The position of the $v_{1}$ vibrational mode depends on the length of the $\pi$-electron conjugated chain in isolated carotenoids ${ }^{30}, 39$. This band occurs at 1530.2 and $1529.1 \mathrm{~cm}^{-1}$ for violaxanthin and lutein in cyclohexane, respectively. The $v_{1}$ frequency in daffodil chromoplasts exhibits a clear dependence on the excitation wavelength, peaking at 1530.4, $1526.7 \& 1528.0 \mathrm{~cm}^{-1}$ for excitation at 488.0, $501.7 \&$ $406.7 \mathrm{~nm}$, respectively (see figure $2 \mathrm{~d}$ ). These different frequencies support the presence of three non-identical carotenoid species - note that the three excitation wavelengths correspond to the absorption peaks of species 1-3 $(487,500 \& 402 \mathrm{~nm}$, respectively). Due to the excitonic nature of aggregates, it is not possible to relate the $v_{1}$ frequency to that of monomeric violaxanthin or lutein. However, the position of the $v_{1}$ mode permits us to confirm the presence of more than one aggregate species with very close absorption.

Around $116 \mathrm{o} \mathrm{cm}^{-1}$, a group of bands termed $v_{2}$ constitutes a fingerprint for the isomerization state (cis/trans) of the carotenoid carbon backbone ${ }^{40-42}$. Chromoplasts display the same modes at all wavelengths used, with a dominant band at $1058 \mathrm{~cm}^{-1}$ and two satellites circa $1188 \& 1214 \mathrm{~cm}^{-1}$. The absence of a vibrational mode at $1130 \mathrm{~cm}^{-1}$ in daffodil chromoplasts, as for the isolated standards, confirms that both lutein and violaxanthin are in the all-trans configuration ${ }^{40-}$ ${ }^{42}$. Observed around $1000 \mathrm{~cm}^{-1}$, the $v_{3}$ band arises from inplane rocking vibrations of the methyl groups attached to the conjugated chain, coupled with in-plane bending modes of the adjacent $\mathrm{C}-\mathrm{H}^{\prime} \mathrm{s}^{43}$. The $v_{3}$ mode is a fingerprint of the conformation of carotenoid conjugated end-cycles ${ }^{44}$. This band can thus be used to track the presence of lutein and/or violaxanthin in the chromoplast spectra, as a result of differences in their end-rings. Lutein in cyclohexane shows a sharp asymmetric band peaking at $1003.1 \mathrm{~cm}^{-1}$ with a small shoulder ca. $1007.9 \mathrm{~cm}^{-1}$, while violaxanthin presents a broad band peaking at $1006.5 \mathrm{~cm}^{-1}$ with a clear shoulder ca. $1002.9 \mathrm{~cm}^{-1}$. Chromoplasts excited at $501.7 \mathrm{~nm}$ exhibit a sharp vibrational mode at 1004.4 $\mathrm{cm}^{-1}$, resembling the spectrum of monomeric lutein, thus indicating that this carotenoid is dominant in species 2. At 488.0 \& 406.7 $\mathrm{nm}$, the $v_{3}$ band is wider with a prominent shoulder extending up to $1007.2 \mathrm{~cm}^{-1}$ - indicating the presence of both violaxanthin and lutein in species 1 \& 3 (see figure 2c). The $v_{4}$ mode around $960 \mathrm{~cm}^{-1}$ arises from $\mathrm{C}-\mathrm{H}$ out-of-plane wagging motions coupled with $\mathrm{C}=\mathrm{C}$ torsional modes, 39 and this region is an indicator of out-of-the-plane distortions of the carotenoid conjugated chain 39 , 45. In chromoplasts, this region contains small but clear modes at 951.8 and $963.8 \mathrm{~cm}^{-1}$, which are also present for isolated lutein and violaxanthin. Thus, all three species appear to experience no significant distortions from their relaxed conformation.
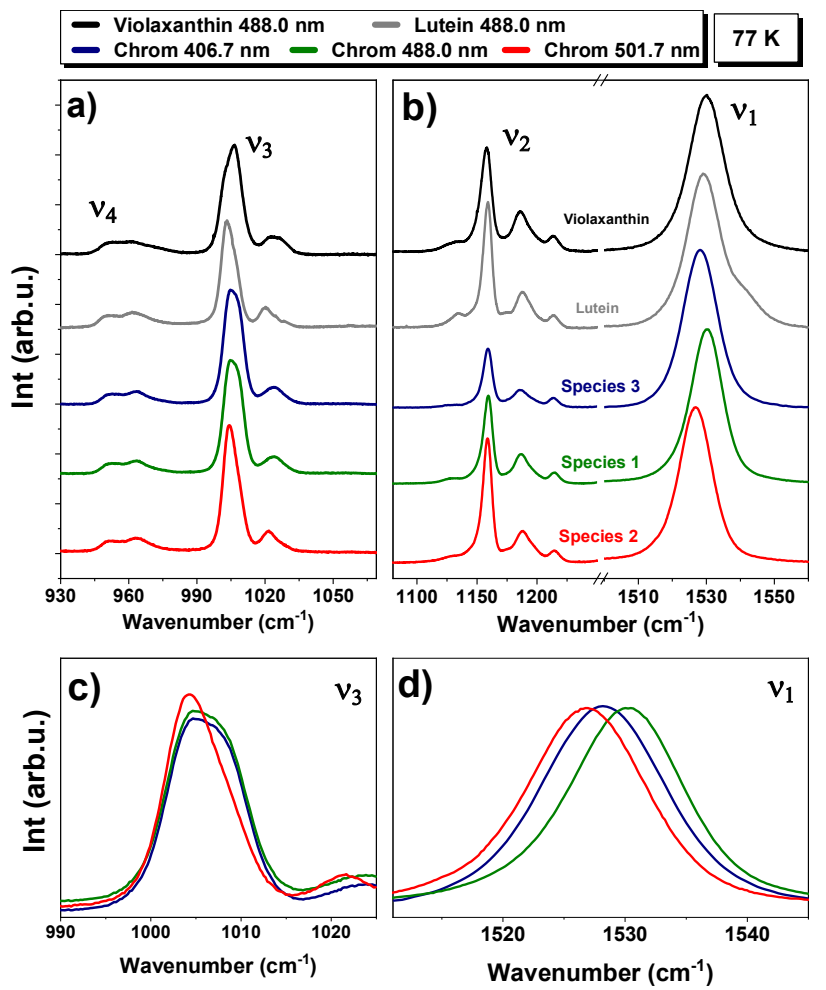

Figure 2: $77 \mathrm{~K}$ resonance Raman spectra of violaxanthin (black) and lutein (grey) in cyclohexane, excited at $488.0 \mathrm{~nm}$, and of intact chromoplasts excited at 406.7, 488.0 \& $501.7 \mathrm{~nm}$ (blue, green, red respectively), showing the 930-1050 and 1100$1600 \mathrm{~cm}^{-1}$ regions (a, b respectively). The $v_{3}$ and $v_{1}$ regions are expanded in panels $\mathrm{c}$ and $\mathrm{d}$, respectively (normalized to peak height).

Figure 3 shows the time-resolved absorption data obtained for chromoplasts in the $100 \mathrm{fs}-5 \mathrm{~ns}$ time window, measured with an excitation pulse centered at $488 \mathrm{~nm}$ to investigate the photochemistry of the major population of carotenoid aggregates (species 1). The dataset overview (Figure 3a) shows that different parts of the ground-state bleaching (GSB) region in the 350-475 $\mathrm{nm}$ range decay with markedly different kinetics, and the excited state absorption (ESA) band in the 500-600 $\mathrm{nm}$ region exhibits two prominent peaks that reach their maxima at different times. Both these trends can be observed in more detail in the kinetic traces, presented in Figure $3 \mathrm{~b}$. The two ESA bands evolve at different rates, with the $552-\mathrm{nm}$ peak appearing and then decaying slightly before the one at 528 $\mathrm{nm}$. The complex evolution of these ESA bands is clearly observed in time-gated spectra (Figure $3 \mathrm{c}$ ). The initial $\mathrm{S}_{2}$ excited state, observed by its negative stimulated emission (SE) band visible above $505 \mathrm{~nm}$ in the 120 -fs spectrum (black trace), relaxes very quickly - evolving into a broad, almost featureless band peaking around $560 \mathrm{~nm}$ (red trace, $300 \mathrm{fs}$ ). This broad feature can be attributed to a vibrationally-hot S1 state. The evolution of this state is different from that observed for isolated carotenoids - instead of 
narrowing to a well-defined S1 ESA peak, a nearly-symmetrical doublet appears with maxima at 528 and $552 \mathrm{~nm}$ (green trace, $2 \mathrm{ps}$ ). These peaks decay at different rates, so that the 528-nm maximum dominates the 15-ps spectrum (blue trace). At later times the ESA band shifts further to the blue, where it partly decays within 100 ps (cyan trace).
However, a small fraction of this signal remains beyond the 5-ns time window of the experiment (eg. pink trace, 2-5 $\mathrm{ns})$. The evolution at longer timescales is again different from that seen for monomeric carotenoids in solution - including violaxanthin and lutein - which return to the ground state in tens of ps ${ }^{12}$.
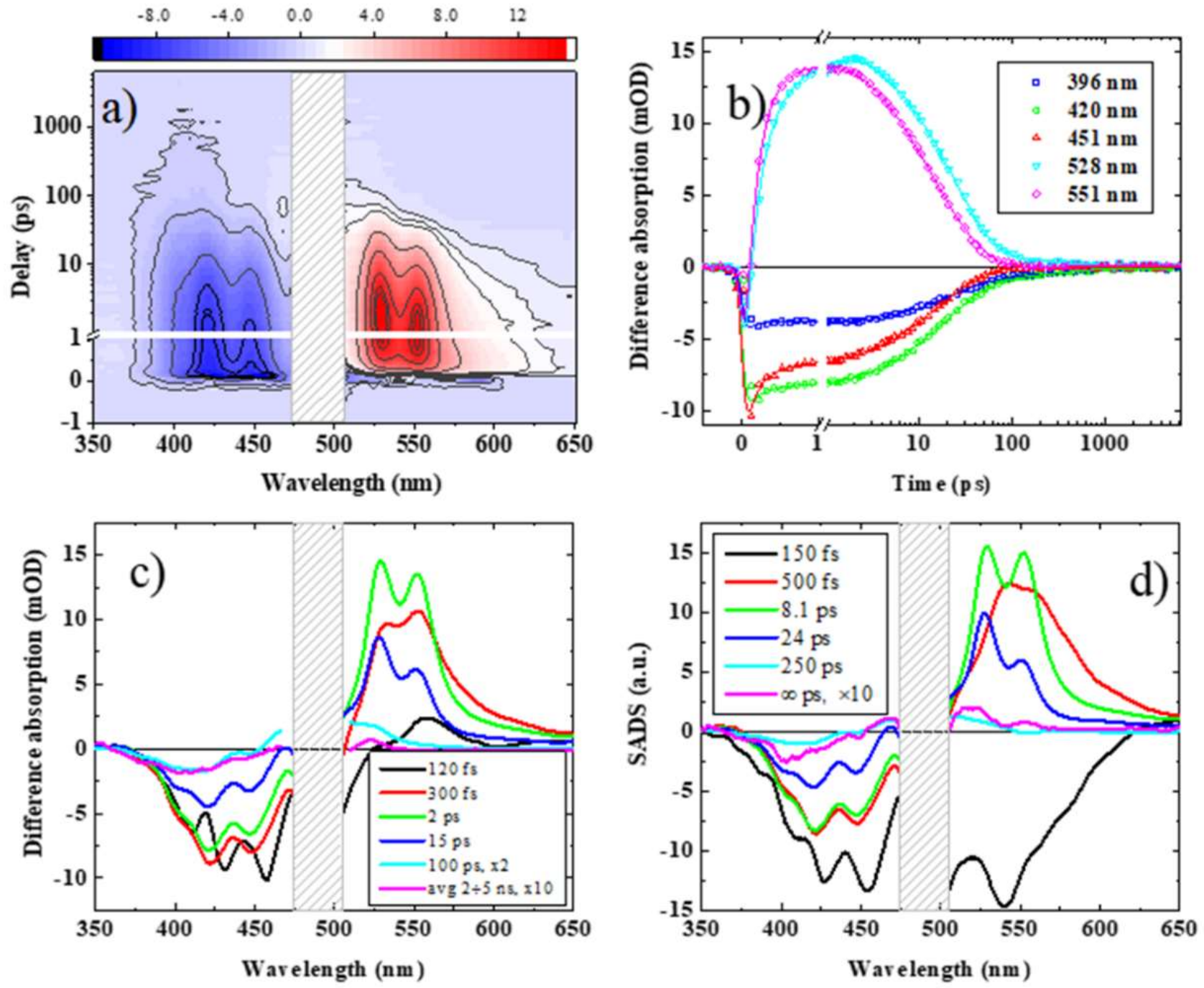

Figure 3: Femtosecond-to-nanosecond transient absorption data of daffodil chromoplasts obtained with excitation at 488 nm: (a) dataset overview; (b) selected absorption-difference kinetic traces (symbols - experimental data; solid lines - results of global fit, as per d); (c) time-gated spectra at selected delay times, as indicated; (d) EADS estimated by global fitting, using 6-component evolutionary model (decay times indicated in the legend). Greyed regions indicate spectral range corrupted by scattering of the pump pulses.

Global analysis using an evolutionary model yields the Evolution-Associated Difference Spectra (EADS) shown in Figure 3d, reflecting a cascade of events with the following time constants: 150 fs $\rightarrow 500$ fs $\rightarrow 8.1 \mathrm{ps} \rightarrow 24 \mathrm{ps} \rightarrow 250 \mathrm{ps}$ $\rightarrow$ non-decaying. These first two EADS at 150 and 500 fs (black, red spectra) are similar to those observed for monomeric carotenoids, reflecting analogous evolution over the same timescales ${ }^{12}$. The first step corresponds to the decay of the $S_{2}$ state ( $150 \mathrm{fs}$, black spectrum), somewhat obscured by cross-phase modulation between pump and probe. The second step corresponds to cooling of the resulting hot $\mathrm{S}_{1}$ state (500 fs, red spectrum), whereby ESA loses its red shoulder at 550-650 $\mathrm{nm}$. The third EADS (8.1 ps, green spectrum) presents two peaks of similar intensity at 528 and $552 \mathrm{~nm}$, while the GSB in the blue part of the spectrum remains unchanged relative to the 500-fs spectrum. In the fourth EADS (24 ps, blue spectrum) a substantial decrease of relative intensity of the $552-\mathrm{nm}$ peak is observed. The fifth EADS (250 ps, cyan spectrum) presents a distorted shape of the GSB on its red wing, being attenuated by about $90 \%$, possibly indicating the presence of a 
transient species with ESA overlapping in this region and pushing the signal up. The peaks at 528 and $552 \mathrm{~nm}$ disappear completely, while a broad ESA band peaks at ca. 507 $\mathrm{nm}$. This sixth EADS (œ, pink spectrum) is similar to that at 250 ps and persists beyond $5 \mathrm{~ns}$ (time window of the experiment). It should be noted that the two excited species with ESA peaking at 528 and $552 \mathrm{~nm}$ decay in parallel, and they cannot be separated in this sequential model. This model is therefore unable to describe the dynamics after photoexcitation completely, while it is adequate to perform an initial assessment of the excited species that will lead to the design of a representative model (see target analysis, below and Fig 5 ). In monomeric carotenoids in solution, $\mathrm{S}_{1}$ decays to the ground state in a few picoseconds, with no long-living species. The ESA position and decay rate for $S_{1}$ in monomeric violaxanthin and lutein are 532 $\mathrm{nm} \& 24 \mathrm{ps}$, and $558 \mathrm{~nm} \& 14 \mathrm{ps}$, respectively ${ }^{12}$. In addition, the GSB intensity for isolated carotenoids decays in proportion to the $\mathrm{S}_{1}$ decay. In daffodil chromoplasts, the unchanged intensity of GSB between 500 fs and 8.1 ps indicates that the recombination of excited states is somewhat compensated by the formation of additional excited species depleting the ground state. It suggests that the two ESA cannot be assigned to independent $\mathrm{S}$ states decaying to the ground state. In the literature, similar ESA doublets have been reported for aggregates of $\beta$-carotene ${ }^{20}$ and zeaxanthin $^{22,23}$. Hence, the red ESA may be assigned to $S_{1}$ (generated by excitonic coupling) decaying in a few ps, while the blue ESA can be assigned to a triplet state decaying over a wide range of timescales, from 1os to 100 of ps. Hence, the ESA observed for daffodil chromoplasts at 528 $\mathrm{nm}$ may indicate the presence of triplet states.

In order to analyze the fate of the excitation at longer timescales, transient absorption spectra of daffodil chromoplasts in the ns-to- $\mu$ s range were measured in buffer solutions saturated with oxygen, air and argon (Fig. 4). All transient absorption kinetics can be fitted to a mono-exponential decay, and the gated spectra for chromoplasts at three different oxygen concentrations are nearly identical. They all show a wide negative region around $350-440 \mathrm{~nm}$, attributable to GSB. In the 440-500 $\mathrm{nm}$ region, the ground state bleaching is mixed up with ESA, with negative contributions at 480 and $444 \mathrm{~nm}$ corresponding to the o-o and o-1 vibronic peaks of the $\mathrm{S}_{0} \rightarrow \mathrm{S}_{2}$ electronic transitions, respectively. The ESA in the region 500-530 $\mathrm{nm}$ exhibits a peak at $505 \mathrm{~nm}$, accompanied by a signal at $520 \mathrm{~nm}$ appearing as a peak in argon or a shoulder in air and oxygen. These signals decay with lifetimes of $180 \mathrm{~ns}, 700 \mathrm{~ns}$, and 3.5 $\mu \mathrm{s}$ in oxygen, air and argon, respectively. The dependence of this lifetime on oxygen concentration (Fig. 4), a molecule known to enhance inter-system crossing in carotenoid triplet states ${ }^{46}, 47$, is consistent with the attribution of these ESA to $T_{1} \rightarrow T_{n}$ transitions. In monomers of the plant antenna protein LHCII, no significant difference is observed between lutein and violaxanthin triplets, in the native protein and the npq1lut2 mutant, respectively $-\mathrm{T}_{1} \rightarrow \mathrm{T}_{\mathrm{n}}$ peaks at $508 \mathrm{~nm}$ in both cases ${ }^{48}$. The weak excitonic coupling of species 1 and 2 may introduce changes in their $T_{1} \rightarrow T_{n}$ ESA position. It therefore seems reasonable to assign the higher-energy $\mathrm{T}_{1} \rightarrow \mathrm{T}_{\mathrm{n}}$ transition at $505 \mathrm{~nm}$ to associated lutein/violaxanthin triplets in species 1 (o-o at $481 \mathrm{~nm}$ a room temperature), while associated lutein (species 2; $500 \mathrm{~nm}$ ) contributes to the lower-energy triplet signal at $520 \mathrm{~nm}$.
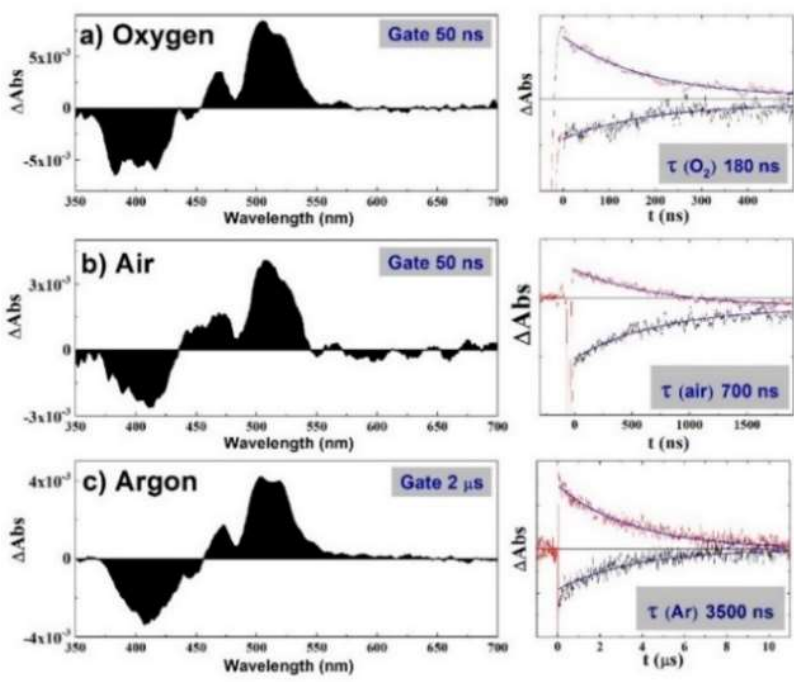

Figure 4: Transient absorption in the ns-to- $\mu$ s window for daffodil chromoplasts upon 488-nm excitation: gated spectra and time evolution for 405 and $500 \mathrm{~nm}$ in (a) oxygen, (b) air, and (c) argon.

The only known process capable of forming triplet states in carotenoids in the absence of a photo-sensitizer is singlet exciton fission. This process has been observed in several cases where intermolecular interactions occur between carotenoids ${ }^{13},{ }^{17-23}$. In acenes, the mechanism of triplet formation is fairly well understood. A singlet-excited state $\left(\mathrm{S}_{1}\right)$ on one pigment shares its energy with a neighboring pigment in the ground state $\left(\mathrm{S}_{\mathrm{o}}\right)$, resulting in a correlated pair of triplets ${ }^{1}(\mathrm{TT})$ on these two molecules, entangled with spin coherence and overall zero spin. This strongly-entangled state has a singlet character, so that its decay to the singlet ground state is allowed with rates reminiscent of singlet excited states (ps-ns) ${ }^{14,15}$. Conversely, the intermediates involved in the evolution of this ${ }^{1}(\mathrm{TT})$ state remain controversial. A weakly-entangled triplet $\left(T_{1} \ldots T_{1}\right)$ is necessary as an intermediate step, which may either yield two independent triplet states $\left(T_{1}+T_{1}\right)$, or recombine by triplet-triplet annihilation. The spectral position of the ${ }^{1}$ (TT) signature is not well established - for acenes it appears blue-shifted with respect to an independent triplet, while it has been proposed as red-shifted for terrylenediimide dimers ${ }^{49}$ and $\beta$-carotene aggregates ${ }^{14,15}$.

In carotenoids, photoexcitation leads to the formation of the second excited state $\left(\mathrm{S}_{2}\right)$, with an energy of the lowest vibrational level at $2.57 \mathrm{eV}$. The $\left(\mathrm{S}_{1}\right)$ singlet state consists of two coupled triplets residing on the same isolated molecule $^{10}$. Consequently, the mechanism of singlet fission in daffodil chromoplasts must take into account the contribution of the second excited state, while also allowing for the excitonic character of $\mathrm{S}_{2}, \mathrm{~S}_{1}$ and $\mathrm{T}_{1}$. Excitation energy is transferred from $S_{2}$ to the higher vibrational levels of the first excited state (hot $S_{1}$ ) in 150 femtoseconds, whereupon two pathways compete in ca. 500 fs: i) decay to the lowest 
vibrational states of $S_{1}$, and ii) formation of an entangled triplet state ${ }^{1}(\mathrm{TT})$ between two neighboring carotenoid molecules, with both triplets residing on the same excitonic manifold. The relaxed $S_{1}$ state decays in 11 ps to the ground state $\left(\mathrm{S}_{\mathrm{o}}\right)$, whereas the entangled triplets ${ }^{1}(\mathrm{TT})$ either decay in 36 ps to the ground state (transition allowed due to singlet character), or dissociate into weakly-entangled and then independent triplets $\left(T_{1} \ldots T_{1} \& T_{1}+T_{1}\right.$, respectively). Target analysis based on this model, presented in Figure 5a, produces a remarkably good fit to the femtosecond data (see solid lines in Fig. 5b), and the resulting spectra exhibit the exact features expected from the model. This is clear from the hot $S_{1}$, the relaxed $S_{1}$ and the ${ }^{1}(T T)$ states, which exhibit very well-separated ESA. Hot $S_{1}$ has a broad and featureless induced absorption with a pronounced red shoulder, the relaxed $S 1$ state exhibits a narrow and well-defined peak at $553 \mathrm{~nm}$, and the ${ }^{1}(\mathrm{TT})$ state peaks at $528 \mathrm{~nm}$. This ${ }^{1}(\mathrm{TT})$ decays following two competitive channels, with a 36-ps decay to the ground state, or towards $\left(T_{1} \ldots T_{1}\right)$ in $240 \mathrm{ps}$. The weakly-entangled triplets $\left(T_{1} \ldots T_{1}\right)$ and independent triplets $\left(T_{1}+T_{1}\right)$ are modeled as two exponentially-decaying states with identical spectra. They show a broad induced absorption band peaking around $510 \mathrm{~nm}$, with no ESA above $550 \mathrm{~nm}$ at long timescales.

This set of experiments additionally allows a fairly precise estimation of the energy of the $T_{1}$ state of lutein/violaxanthin aggregates, a parameter which has never been measured directly. The energy of the $\mathrm{S}_{2}$ state is $2.57 \mathrm{eV}$, as obtained from the absorption spectrum, and that of the relaxed $\mathrm{S}_{1}$ state has been estimated at $1.55 \mathrm{eV}^{50}$. The energy level of ${ }^{1}(T T)$ can be assumed to be equal to $S_{1}$ within a difference depending on the entanglement energy $(0.15 \mathrm{eV})^{14}$, ${ }^{15}$, while the independent triplets must obey the energetic condition $E\left(S_{1}\right) \geq 2 \bullet E\left(T_{1}\right)$. Hence the ${ }^{1}(T T)$ energy is $1.55 \pm 0.20 \mathrm{eV}$, while the $T_{1}$ energy level for aggregated carotenoids in daffodils is $0.78 \pm 0.10 \mathrm{eV}$ (Scheme 1).
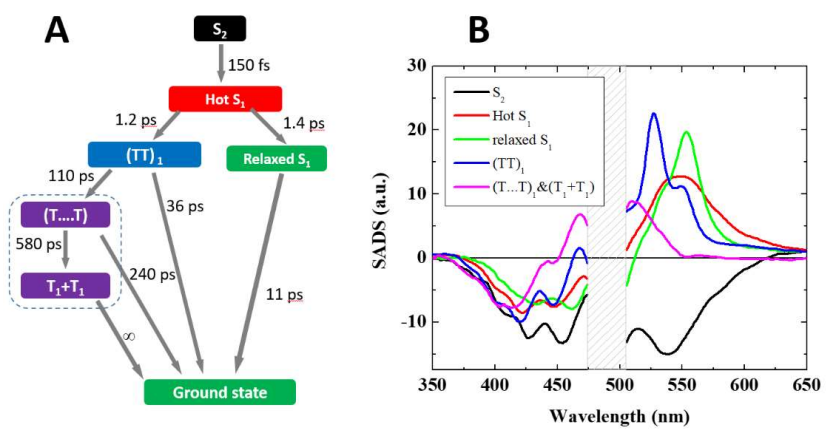

Figure 5: Target model (a) and species-associated difference spectra (b) resulting from target analysis of femtosecond pump-probe data on daffodil chromoplasts. The estimated time constants are shown next to the corresponding arrows in panel a.

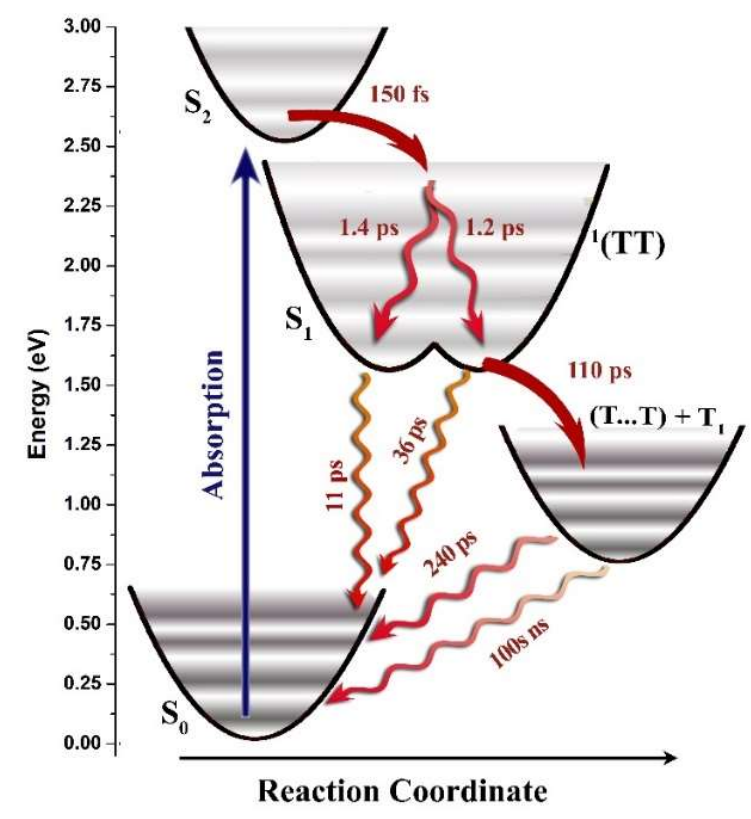

Scheme 1: Energetic pathways in aggregated lutein \& violaxanthin after absorption of one photon (lifetimes as obtained from target analysis).

\section{CONCLUSIONS}

In daffodil chromoplasts, the interactions of lutein and violaxanthin lead to the existence of three different species of carotenoids aggregates, the most abundant being a mixture of lutein/violaxanthin with o-o transition at $481 \mathrm{~nm}$. The photodynamics of this species induces the emergence of $\mu$ s-lived triplet states produced via singlet fission, as already observed for self-associated lycopene in ripe tomato fruit. This first report of singlet fission occurring in mature flower plastids suggests that triplet fission is a phenomenon general to carotenoid aggregates in chromoplasts. While in tomatoes, lycopene interactions induce both a large redshift and strong distortions of the lycopene molecule $^{13}$, in daffodil chromoplasts singlet fission is observed from lutein/violaxanthin aggregates which exhibit only a moderate red-shift in absorption transitions, and no molecular distortion of the interacting carotenoids. The fact that we observe singlet fission in both fruits and, for the first time here, flowers, occurring in carotenoid assemblies displaying different organizations and levels of interaction (strong and medium-to-weak, respectively), suggests a specific role of singlet fission in plastids. Finally, the structural organization of lutein/violaxanthin in daffodil chromoplasts produces well-defined electronic levels, allowing the energetic pathways following carotenoid singlet fission to be disentangled unequivocally for the first time.

\section{ASSOCIATED CONTENT}

Supporting Information. HPLC determination of carotenoid content in daffodil chromoplasts (Fig. S1); linear combination of in vitro absorption spectra of isolated carotenoids, to reproduce the spectrum of solubilized chromoplasts (Fig. S2). This material is available free of charge via the Internet at http://pubs.acs.org. 


\section{AUTHOR INFORMATION}

\section{Corresponding Author}

* Mikas Vengris, Laser Research Center, Faculty of Physics, Vilnius University, Saulètekio Ave. 10, LT-10223, Vilnius, Lithuania.

e-mail: mikas.vengris@ff.vu.lt

* Manuel J Llansola-Portoles, Université Paris-Saclay, CEA, CNRS, Institute for Integrative Biology of the Cell (I2BC), 91198, Gif-sur-Yvette, France.

e-mail: manuel.llansola@izbc.paris-saclay.fr

\section{Author Contributions}

The manuscript was written through contributions of all authors.

\section{ACKNOWLEDGMENTS}

This work was supported by the French Infrastructure for Integrated Structural Biology (FRISBI) ANR-10-INBS-05, and the CNRS as part of its 8oPRIME interdisciplinary program. M.V. was supported by the European Regional Development Fund according to the supported activity 'Research Projects Implemented by World-class Researcher Groups' under Measure No. 01.2.2-LMT-K-718, grant number 01.2.2-LMT-K-718-01oo14. A.K.L. and the IJPB are supported by Saclay Plant Sciences-SPS (ANR-17-EUR-ooo7). This work benefited from the support of IJPB's Plant Observatory technological platforms.

\section{REFERENCES}

1. I. Egea, C. Barsan, W. Bian, E. Purgatto, A. Latché, C. Chervin, M. Bouzayen and J.-C. Pech, Plant Cell Physiol., 2010, 51, 16011611.

2. L. Li and H. Yuan, Arch. Biochem. Biophys., 2013, 539, 102-109.

3. K. J. v. Wijk and F. Kessler, Annu. Rev. Plant Biol., 2017, 68, 253289.

4. L. U. Hansen and M.-C. M. Chiu, J. Agric. Food Chem., 2005, 53, 6678-6682.

5. E. Lewinsohn, Y. Sitrit, E. Bar, Y. Azulay, M. Ibdah, A. Meir, E. Yosef, D. Zamir and Y. Tadmor, Trends Food Sci. Technol., 2005, 16, 407-415.

6. G. Britton, S. Liaaen-Jensen and H. Pfander, Carotenoids, Volume 4: Natural Functions, Birkhäuser Verlag, Switzerland, 2008.

7. P. Jahns and A. R. Holzwarth, Biochim. Biophys. Acta, Bioenerg., 2012, 1817, 182-193.

8. A. V. Ruban, R. Berera, C. Ilioaia, I. H. M. van Stokkum, J. T. M. Kennis, A. A. Pascal, H. van Amerongen, B. Robert, P. Horton and R. van Grondelle, Nature, 2007, 450, 575-578.

9. M. P. Johnson, M. Havaux, C. Triantaphylidès, B. Ksas, A. A. Pascal, B. Robert, P. A. Davison, A. V. Ruban and P. Horton, J. Biol. Chem., 2007, 282, 22605-22618.

10. P. Tavan and K. Schulten, Physical Review B, 1987, 36, 43374358.

11. H. A. Frank, A. J. Young, G. Britton and R. J. Cogdell, Kluwer Academic Publishing, 1999.
12. D. M. Niedzwiedzki, J. O. Sullivan, T. Polívka, R. R. Birge and H. A. Frank, J. Phys. Chem. B, 2006, 110, 22872-22885.

13. M. J. Llansola-Portoles, K. Redeckas, S. Streckaité, C. Ilioaia, A. A. Pascal, A. Telfer, M. Vengris, L. Valkunas and B. Robert, Phys. Chem. Chem. Phys., 2018, 20, 8640 - 8646.

14. A. J. Musser and J. Clark, Annu. Rev. Phys. Chem., 2019, 70, 323-351.

15. K. Miyata, F. S. Conrad-Burton, F. L. Geyer and X. Y. Zhu, Chem. Rev. (Washington, DC, U. S.), 2019, 119, 4261-4292.

16. M. B. Smith and J. Michl, Chem. Rev. (Washington, DC, U. S.), 2010, 110, 6891-6936.

17. A. J. Musser, M. Maiuri, D. Brida, G. Cerullo, R. H. Friend and J. Clark, J. Am. Chem. Soc., 2015, 137, 5130-5139.

18. M. Fuciman, M. Durchan, V. Šlouf, G. Keşan and T. Polívka, Chem. Phys. Lett., 2013, 568-569, 21-25.

19. H.-T. Chang, Y.-Q. Chang, R.-M. Han, P. Wang, J.-P. Zhang and L. H. Skibsted, J. Agric. Food Chem., 2017, 65, 6058-6062.

20. D. Zhang, L. Tan, J. Dong, J. Yi, P. Wang and J. Zhang, Chem. Res. Chin. Univ., 2018, 34, 643-648.

21. C. Wang and M. J. Tauber, J. Am. Chem. Soc., 2010, 132, 1398813991.

22. C. Wang, M. Angelella, C.-H. Kuo and M. J. Tauber, Proceedings of SPIE, 2012, 8459, 05-13.

23. H. H. Billsten, V. Sundström and T. Polívka, J. Phys. Chem. A, 2005, 109, 1521-1529.

24. B. Camara, P. Hugueney, F. Bouvier, M. Kuntz and R. Monéger, in International Review of Cytology, eds. K. W. Jeon and J. Jarvik, Academic Press, 1995, vol. 163, pp. 175-247.

25. B. Liedvogel, P. Sitte and H. Falk, Cytobiologie 1976, 12, 155 174.

26. S. Al-Babili, J. v. Lintig, H. Haubruck and P. Beyer, The Plant Journal, 1996, 9, 601-612.

27. M. Grabsztunowicz, P. Mulo, F. Baymann, R. Mutoh, G. Kurisu, P. Sétif, P. Beyer and A. Krieger-Liszkay, The Plant Journal, 2019, 99, 245-256.

28. K. Redeckas, V. Voiciuk, R. Steponavičiūtė, V. Martynaitis, A. Šačkus and M. Vengris, J. Phys. Chem. A, 2014, 118, 5642-5651.

29. M. J. Llansola-Portoles, C. Uragami, A. A. Pascal, D. Bina, R. Litvin and B. Robert, Biochim. Biophys. Acta, Bioenerg., 2016, 1857, 1759-1765.

30. M. M. Mendes-Pinto, E. Sansiaume, H. Hashimoto, A. A. Pascal, A. Gall and B. Robert, J. Phys. Chem. B, 2013, 117, 11015-11021.

31. F. C. Spano, J. Am. Chem. Soc., 2009, 131, 4267-4278.

32. S. Akimoto, I. Yamazaki, T. Sakawa and M. Mimuro, J. Phys. Chem. A, 2002, 106, 2237-2243.

33. M. K. Shuklaa, M. J. Llansola-Portoles, M. Tichý, A. A. Pascal, B. Robert and R. Sobotka, Photosynth. Res., 2017, 137, 29-39.

34. M. Kasha, Radiat. Res., 1963, 20, 55-70.

35. S. Köhn, H. Kolbe, M. Korger, C. Köpsel, B. Mayer, H. Auweter, E. Lüddecke, H. Bettermann and H.-D. Martin, in Carotenoids: 
Volume 4: Natural Functions, eds. G. Britton, S. Liaaen-Jensen and H. Pfander, Birkhäuser Basel, Basel, 2008, DOI: 10.1007/978-3-7643-7499-0_5, pp. 53-98.

36. C. Wang, C. J. Berg, C.-C. Hsu, B. A. Merrill and M. J. Tauber, J. Phys. Chem. B, 2012, 116, 10617-10630.

37. G. Zajac, E. Machalska, A. Kaczor, J. Kessler, P. Bouř and M. Baranska, Phys. Chem. Chem. Phys., 2018, 20, 18038-18046.

38. B. Robert, Photosynth. Res., 2009, 101, 147-155.

39. L. Rimai, M. E. Heyde and D. Gill, J. Am. Chem. Soc., 1973, 95, 4493-4501.

40. Y. Koyama, I. Takatsuka, M. Nakata and M. Tasumi, J. Raman Spectrosc., 1988, 19, 37-49.

41. Y. Koyama, T. Takii, K. Saiki and K. Tsukida, Photobiochem. Photobiophys., 1983, 5, 139-150.

42. Y. Koyama, M. Kito, T. Takii, K. Saiki, K. Tsukida and J. Yamashita, Biochim. Biophys. Acta, Bioenerg., 1982, 680, 109118.
43. S. Saito and M. Tasumi, J. Raman Spectrosc., 1983, 14, 310 321.

44. M. M. Mendes-Pinto, D. Galzerano, A. Telfer, A. A. Pascal, B. Robert and C. Ilioaia, J. Biol. Chem., 2013, 288, 18758-18765.

45. M. Lutz, W. Szponarski, G. Berger, B. Robert and J.-M. Neumann Biochem. Biophys. Acta, 1987, 894, 423-433.

46. D. Siefermann-Harms and A. Angerhofer, Photosynth. Res., 1998, 55, 83-94.

47. T. O. Truscott, E. J. Land and A. Sykes, Photochem. Photobiol., 1973, 17, 43-51.

48. F. Saccon, M. Durchan, R. Kaňa, O. Prášil, A. V. Ruban and T. Polívka, J. Phys. Chem. B, 2019, 123, 9312-9320.

49. E. A. Margulies, C. E. Miller, Y. Wu, L. Ma, G. C. Schatz, R. M. Young and M. R. Wasielewski, Nat Chem, 2016, 8, 1120-1125.

50. T. Polívka and V. Sundström, Chem. Phys. Lett., 2009, 477, 111. 